\title{
Relationships between Otolith Size and Body Size for Hawaiian Reef Fishes ${ }^{1}$
}

\author{
Ken Longenecker ${ }^{2}$
}

\begin{abstract}
Estimating body size of fishes from remains recovered from piscivores, archaeological sites, and sedimentary deposits is desirable but rarely accomplished because the relationships between the size of a fish and its durable anatomical structures are largely unknown. Regression equations to predict the size or weight of 41 common Hawaiian reef fishes from sagittae (saccular otoliths) are presented. Data are also grouped into higher taxa to permit size predictions when otoliths cannot be assigned to species.
\end{abstract}

Animal remains are frequently used to reconstruct faunal assemblages in dietary analysis, archaeology, geology, and paleontology. This work on fishes is difficult because bones and scales can be hard to identify, their numbers may vary among individuals, and the relationship between the size of a bone or scale and the size of the fish that produced it may be unknown.

Otoliths, particularly sagittae or saccular otoliths, may be used to circumvent these problems because they are taxonomically distinct and the number per individual does not vary. Further, they are harder and more durable than skeletal components and are often

\footnotetext{
1 This paper is funded in part by a grant/cooperative agreement from the National Oceanic and Atmospheric Administration, Project no. R/FM-8, which is sponsored by the University of Hawai'i Sea Grant College Program, SOEST, under Institutional Grants No. NA86RG0041 and NA16RG2254 from NOAA Office of Sea Grant, Department of Commerce. The views expressed herein are those of the author and do not necessarily reflect the views of NOAA or any of its subagencies. UNIHISEAGRANT-JC-02-32. NOAA Fisheries, Pacific Islands Fisheries Science Center supported preparation of the manuscript. This is Hawai'i Institute of Marine Biology contribution no. 1297 and contribution 2007-014 of the Hawai'i Biological Survey. Manuscript accepted 28 November 2007.

${ }^{2}$ Hawai'i Institute of Marine Biology, P.O. Box 1346, Kāne'ohe, Hawai'i 96744. Current address: Bishop Museum, 1525 Bernice Street, Honolulu, Hawai'i 96817 (e-mail: klongenecker@bishopmuseum.org).
}

Pacific Science (2008), vol. 62, no. 4:533-539

(C) 2008 by University of Hawai'i Press

All rights reserved the only identifiable remains found in geological strata (Rivaton and Bourret 1999). Otoliths are common fossils throughout broad geographic and stratigraphic ranges (Hecht 1990), may be the only evidence that a fish species was present at archaeological sites (Weisler 2002), and are often the only identifiable fish remains found in the stomachs or feces of predators (Hecht 1990). Finally, the size of otoliths can be used to estimate fish size, and a fair number of these relationships have been described for fishes worldwide (Frost and Lowry 1981, Echevierria 1987, Gamboa 1991, Plötz et al. 1991, Smale et al. 1995, Granadeiro and Silva 2000, Harvey et al. 2000, Mikkelsen et al. 2002, Naya et al. 2002, Waessle et al. 2003).

No large-scale analysis of otolith-fish size relationships has been conducted for Hawaiian fishes. With 1,250 species of which $22.3 \%$ of coastal species are endemic (Mundy 2005), this information is needed for research on resource use by early Hawaiians, dietary analysis of piscivores, and reconstruction of ancient marine environments. Here I present analyses of the relationship between otolith length and fish length or weight. These results complement a guide to the identification of Hawaiian fish otoliths (Dye and Longenecker 2004).

\section{MATERIALS AND METHODS}

Fishes were collected from May 2000 through September 2002 from the forereef of Kāne'ohe Bay. Collecting area boundaries were the $5.5 \mathrm{~m}$ isobath shoreward (parallel to 
the barrier reef), the $30.5 \mathrm{~m}$ isobath seaward (the bottom of a drop-off at the mouth of the bay), and boating channels laterally. All specimens were identified, then standard length (in millimeters) and total body weight (in grams) were measured. Sagittae were removed and stored dry in tissue culture plates, with one species per plate and one individual per well. One otolith per fish was haphazardly chosen (or the lone otolith if one was broken or missing) and measured with digital calipers along the rostrum to postrostrum axis (nomenclature of Secor et al. [1992]).

Least squares regression analysis of otolith length versus standard length and otolith length versus total body weight was performed for each species. Data were also combined to search for the same relationships within all higher taxa. Equations are presented only for regressions with $P<.5$.

\section{RESULTS AND DISCUSSION}

Standard length can be modeled as a linear function of otolith length (Appendix 1), and total body weight can be predicted using a power function of otolith length (Appendix 2). For all but two cases, the otolithweight relationships could be modeled with two parameters. The exceptions were threeparameter power functions.

Both appendixes are organized phylogenetically. Species included in supraspecific analyses are either annotated at the end of the appendix (for space considerations) or listed parenthetically adjacent to taxon name if no significant relationship was found for that species alone. Species with significant otolith-fish size relationships are listed along with their regression equations below the supraspecific taxon heading. For example, results for the length regression of the subfamily Pomacentrinae (Appendix 1) include four species: Stegastes marginatus, which did not yield a significant regression on its own, listed parenthetically beside the subfamily name; Abudefduf abdominalis, which did have a significant relationship, listed below the subfamily name; and Plectroglyphidodon imparipennis and $P$. jobnstonianus, indicated by an annotation to the generic name. A significant regression could not be constructed for either species alone; however, a genus-level relationship was significant.

This phylogenetic grouping serves three purposes. First, it can be used to predict the size of a fish from otoliths larger or smaller than those used in the analyses. Although linear relationships with high coefficients of determination, such as many of those in Appendix 1, might reasonably be used for extrapolation, doing so with curvilinear relationships (Appendix 2) is likely to provide unrealistic estimates. Using a relationship for a higher taxon, based on a wider size range of individuals, may help avoid the need for extrapolation. Second, because otoliths are more easily assigned to higher taxa than to species, the groupings provide reasonable predictions of fish size when a species-level identification of an otolith is not feasible. The more-general higher-taxa regressions should be used in such cases. Third, these groups also provide predictions for species not included in the analysis. With 1,250 fishes known from Hawai' $i$, the equations presented here represent just a fraction of the work necessary for detailed reconstruction of fish assemblages. In the interim, these higher-taxa relationships may suffice for predicting fish sizes.

\section{ACKNOWLEDGMENTS}

I thank Annmarie Dehn, Ross Langston, and Joanna Philippoff for donating or helping collect many of the specimens; and Klahzia Longenecker for assisting with otolith extractions.

\section{Literature Cited}

Dye, T. S., and K. R. Longenecker. 2004. Manual of Hawaiian fish remains identification based on the skeletal reference collection of Alan C. Ziegler and including otoliths. Society for Hawaiian Archaeology, Honolulu.

Echevierria, T. W. 1987. Relationship of oto- 
lith length to total length in rockfishes from northern and central California. Fish. Bull. 85:383-387.

Frost, K. J., and L. F. Lowry. 1981. Trophic importance of some marine gadids in northern Alaska and their body-otolith size relationships. Fish. Bull. 79:187-192.

Gamboa, D. A. 1991. Otolith size versus weight and body-length relationships for eleven fish species of Baja California, Mexico. Fish. Bull. 89:701-706.

Granadeiro, J. P., and M. A. Silva. 2000. The use of otoliths and vertebrae in the identification and size-estimation of fish in predator-prey studies. Cybium 24:383393.

Harvey, J. T., T. R. Loughlin, M. A. Perez, and D. S. Oxman. 2000. Relationship between fish size and otolith length for 63 species of fishes from the eastern North Pacific Ocean. NOAA Tech. Rep. NMFS 150.

Hecht, T. 1990. Otoliths: An introduction to their morphology and use in the identification of Southern Ocean fishes. Pages 64-69 in O. Gon and P. C. Heemstra, eds. Fishes of the Southern Ocean. J. L. B. Smith Institute of Ichthyology, Grahamstown, South Africa.

Mikkelsen, B., T. Haug, and K. T. Nilssen. 2002. Summer diet of grey seals (Halichoerus grypus) in Faroese waters. Sarsia 87:462-471.

Mundy, B. C. 2005. Checklist of the fishes of the Hawaiian Archipelago. Bishop Museum Press, Honolulu.
Naya, D. E., M. Arim, and R. Vargas. 2002. Diet of South American fur seals (Arctocephalus australis) in Isla de Lobos, Uruguay. Mar. Mamm. Sci. 18:734-745.

Plötz, J., W. Ekau, and P. J. H. Reijnders. 1991. Diet of Weddell seals Leptonychotes weddellii at Vestkapp, eastern Weddell Sea (Antarctica), in relation to local food supply. Mar. Mamm. Sci. 7:136-144.

Rivaton, J., and P. Bourret. 1999. Les otolithes des poissons de l'Indo-Pacifique. Institut de Recherche pour le Développement, Nouméa.

Secor, D., J. Dean, and E. H. Laban. 1992. Otolith removal and preparation for microstructural examination. Pages 19-57 in D. K. Stevenson and S. E. Campana, eds. Otolith microstructure examination and analysis. Can. Spec. Publ. Fish. Aquat. Sci. 117.

Smale, M. J., G. Watson, and T. Hecht. 1995. Otolith atlas of southern African marine fishes. J. L. B. Smith Institute of Ichthyology, Grahamstown, South Africa.

Waessle, J. A., C. A. Lasta, and M. Favero. 2003. Otolith morphology and body size relationships for juvenile Scianidae in the Rio de la Plata estuary $\left(35-36^{\circ} \mathrm{S}\right)$. Sci. Mar. 67:233-240.

Weisler, M. I. 2002. New fish records from Oundjo (Site 26), La Grande Terre: Further contributions of otoliths. Pages 207211 in S. Bedford, C. Sand, and D. Burley, eds. Fifty years in the field: Essays in honour and celebration of Richard Shutler Jr.'s archaeological career. New Zealand Archaeological Association, Wellington.

Appendix 1

Results of Linear Regression of Otolith Length (OL) versus Standard Length (SL), in millimeters [SL $=a+b(\mathrm{OL})]$

\begin{tabular}{|c|c|c|c|c|c|}
\hline Taxon & $n$ & Range (mm) & $a$ & $b$ & $r^{2}$ \\
\hline \multicolumn{6}{|l|}{ Anguilliformes } \\
\hline \multicolumn{6}{|l|}{ Muraenidae } \\
\hline Gymnothorax ${ }^{a}$ & 17 & $1.48-6.03$ & -152.2564 & 191.6527 & 0.89 \\
\hline \multicolumn{6}{|l|}{ Aulopiformes } \\
\hline \multicolumn{6}{|l|}{ Synodontidae } \\
\hline Synodus ${ }^{b}$ & 4 & $0.62-1.09$ & -8.1623 & 54.6525 & 0.96 \\
\hline \multicolumn{6}{|l|}{ Beryciformes } \\
\hline Holocentridae (Myripristis berndti) & 27 & $3.95-9.66$ & 58.3229 & 11.2756 & 0.77 \\
\hline Sargocentron & 16 & $3.95-8.85$ & 13.4202 & 20.4562 & 0.81 \\
\hline Myripristis kuntee & 8 & $7.34-9.66$ & 80.4908 & 8.5006 & 0.57 \\
\hline
\end{tabular}


Appendix 1 (continued)

\begin{tabular}{|c|c|c|c|c|c|}
\hline Taxon & $n$ & Range (mm) & $a$ & $b$ & $r^{2}$ \\
\hline \multicolumn{6}{|l|}{ Gasterosteiformes } \\
\hline Syngnathoidei (Fistularia commersonii) & 15 & $1.76-4.44$ & 21.3819 & 185.1548 & 0.74 \\
\hline Aulostomus chinensis & 13 & $1.76-2.47$ & -139.8717 & 264.7553 & 0.53 \\
\hline \multicolumn{6}{|l|}{ Scorpaeniformes } \\
\hline \multicolumn{6}{|l|}{ Scorpaenidae } \\
\hline Scorpaenodes (birsutus) & 34 & $1.17-3.00$ & -0.9014 & 13.3501 & 0.91 \\
\hline kelloggi & 28 & $1.17-2.47$ & -1.1135 & 13.5516 & 0.95 \\
\hline Sebastapistes & 34 & $1.30-4.22$ & -7.1377 & 18.7071 & 0.99 \\
\hline coniorta & 18 & $2.52-4.22$ & -13.4726 & 20.9575 & 0.94 \\
\hline fowleri & 6 & $1.40-1.71$ & -3.4567 & 16.0512 & 0.99 \\
\hline galactacma & 10 & $1.30-2.86$ & -4.0268 & 17.0121 & 0.99 \\
\hline \multicolumn{6}{|l|}{ Perciformes } \\
\hline \multicolumn{6}{|l|}{ Cirrhitoidei } \\
\hline Cirrhitidae (Cirrbitops fasciatus) & 89 & $1.70-7.17$ & -28.1050 & 33.5819 & 0.96 \\
\hline Amblycirrbitus bimacula & 18 & $1.70-2.78$ & -12.4234 & 27.1491 & 0.95 \\
\hline Cirrbitus pinnulatus & 23 & $4.01-7.17$ & -39.9257 & 36.7757 & 0.90 \\
\hline Paracirrbites & 43 & $1.74-5.32$ & -35.1914 & 35.2619 & 0.94 \\
\hline arcatus & 18 & $1.74-3.88$ & -22.9247 & 30.0187 & 0.98 \\
\hline forsteri & 25 & $2.41-5.32$ & -40.1079 & 37.5093 & 0.90 \\
\hline \multicolumn{6}{|l|}{ Percoidei } \\
\hline \multicolumn{6}{|l|}{ Apogonidae } \\
\hline Apogoninae & 11 & $1.34-8.00$ & -6.8884 & 14.5747 & 0.94 \\
\hline Apogon erythrinus & 7 & $1.34-3.56$ & -1.2863 & 11.5146 & 0.99 \\
\hline Pristiapogon (kallopterus, taeniopterus) & 4 & $3.78-8.00$ & -40.1918 & 22.5921 & 1.00 \\
\hline \multicolumn{6}{|l|}{ Serranidae } \\
\hline Cephalopholis argus & 67 & $6.02-10.34$ & -192.4328 & 58.6273 & 0.85 \\
\hline Plectranthias nanus & 6 & $1.86-2.70$ & 7.4999 & 10.5065 & 0.99 \\
\hline Pseudogramma polyacanthum & 9 & $0.88-2.96$ & -0.7907 & 18.1518 & 0.99 \\
\hline Carangidae $^{d}$ & 7 & $1.56-13.31$ & 144.2950 & 36.0742 & 0.78 \\
\hline \multicolumn{6}{|l|}{ Lutjanidae } \\
\hline Lutjanus kasmira & 16 & $7.58-9.97$ & 87.3414 & 11.1229 & 0.57 \\
\hline Mullidae & 74 & $2.27-5.14$ & -78.1329 & 71.6053 & 0.90 \\
\hline Mulloidichthys (flavolineatus, vanicolensis) & 4 & $3.12-4.74$ & -186.6056 & 94.5660 & 0.99 \\
\hline Parupeneus (insularis) & 70 & $2.27-5.14$ & -75.7908 & 71.3657 & 0.92 \\
\hline cyclostomus & 9 & $2.37-5.14$ & -94.5359 & 78.5276 & 0.97 \\
\hline multifasciatus & 33 & $2.48-4.13$ & -67.5846 & 69.2349 & 0.91 \\
\hline pleurostigma & 7 & $2.27-4.17$ & -112.3948 & 87.3023 & 0.93 \\
\hline porphyreus & 18 & $2.41-4.63$ & -84.3878 & 71.4596 & 0.97 \\
\hline Chaetodontidae ${ }^{e}$ & 40 & $2.32-5.43$ & -21.7420 & 33.1154 & 0.79 \\
\hline Chaetodon $f$ & 31 & $3.29-5.43$ & -9.2852 & 29.1519 & 0.87 \\
\hline multicinctus & 8 & $3.33-4.01$ & 25.0728 & 18.8563 & 0.76 \\
\hline \multicolumn{6}{|l|}{ Labroidei } \\
\hline \multicolumn{6}{|l|}{ Labridae } \\
\hline Bodianus albotaeniatus & 32 & $2.34-8.32$ & -28.3960 & 41.3056 & 0.96 \\
\hline Cheilininaeg & 4 & $0.83-2.89$ & -3.5213 & 27.0441 & 0.99 \\
\hline Corinae $^{b}$ & 70 & $0.46-5.06$ & -15.6196 & 50.4776 & 0.95 \\
\hline Anampses cuvier & 6 & $2.57-3.84$ & -58.8524 & 77.1388 & 0.99 \\
\hline Coris ( flavovittata, gaimard) & 15 & $1.56-4.43$ & -47.5676 & 66.9298 & 0.91 \\
\hline venusta & 11 & $1.69-2.26$ & -59.0002 & 72.2014 & 0.81 \\
\hline Thalasomma & 43 & $0.46-5.06$ & -12.6037 & 49.2495 & 0.97 \\
\hline ballieui & 23 & $2.06-5.05$ & -13.7798 & 54.7471 & 0.92 \\
\hline duperrey & 19 & $0.46-3.53$ & -7.4138 & 42.1491 & 0.99 \\
\hline Pomacentridae & 53 & $1.24-6.75$ & -13.3273 & 24.5819 & 0.92 \\
\hline Chrominae & 36 & $1.24-6.75$ & -10.9624 & 23.1550 & 0.94 \\
\hline Chromis (hanui, ovalis) & 25 & $1.24-6.75$ & -5.5540 & 20.1414 & 0.91 \\
\hline vanderbilti & 19 & $1.24-2.47$ & -2.6341 & 19.1729 & 0.93 \\
\hline
\end{tabular}


Appendix 1 (continued)

\begin{tabular}{|c|c|c|c|c|c|}
\hline Taxon & $n$ & Range (mm) & $a$ & $b$ & $r^{2}$ \\
\hline Dascyllus albisella & 11 & $3.57-4.07$ & -30.1740 & 29.9936 & 0.59 \\
\hline Pomacentrinae (Stegastes marginatus) & 17 & $1.76-5.64$ & -14.5262 & 26.2209 & 0.89 \\
\hline Abudefduf abdominalis & 8 & $4.66-5.64$ & -2.1212 & 27.4818 & 0.73 \\
\hline Plectroglyphidodon ${ }^{i}$ & 6 & $1.76-3.41$ & 9.0694 & 16.2063 & 0.84 \\
\hline \multicolumn{6}{|l|}{ Scaridae } \\
\hline Cblorurus (perspicillatus, spilurus) & 5 & $4.63-8.40$ & -172.2770 & 74.4305 & 0.95 \\
\hline \multicolumn{6}{|l|}{ Trachinoidei } \\
\hline \multicolumn{6}{|l|}{ Pinguipedidae } \\
\hline Parapercis schauinslandii & 8 & $2.79-3.68$ & 1.6896 & 22.3855 & 0.93 \\
\hline Blennioidei $^{j}$ & 119 & $0.23-2.09$ & 7.5063 & 14.6156 & 0.47 \\
\hline \multicolumn{6}{|l|}{ Blenniidae } \\
\hline Salariini (Exallias brevis, Cirripectes quagga) & 57 & $0.41-2.09$ & 3.6530 & 27.6834 & 0.95 \\
\hline Cirripectes vanderbilti & 22 & $0.79-2.09$ & -1.2033 & 30.0014 & 0.98 \\
\hline Entomacrodus strasburgi & 32 & $0.41-0.85$ & 0.3729 & 33.7774 & 0.93 \\
\hline \multicolumn{6}{|l|}{ Tripterygiidae } \\
\hline Enneapterygius atriceps & 61 & $0.30-1.45$ & 5.6105 & 13.5322 & 0.94 \\
\hline \multicolumn{6}{|l|}{ Gobioidei } \\
\hline \multicolumn{6}{|l|}{ Gobiidae } \\
\hline Gobiinae (Coryphopterus duospilus) & 128 & $0.26-2.55$ & 4.8242 & 14.9124 & 0.73 \\
\hline Eviota (susanae) & 86 & $0.26-0.62$ & 0.7472 & 24.4057 & 0.77 \\
\hline epiphanes & 79 & $0.26-0.62$ & 0.6536 & 24.5277 & 0.77 \\
\hline Priolepis & 11 & $0.45-0.97$ & 2.5390 & 14.7486 & 0.90 \\
\hline eugenius & 6 & $0.45-0.96$ & 3.2763 & 13.7504 & 0.84 \\
\hline farcimen & 5 & $0.54-0.97$ & 1.7377 & 15.8309 & 0.97 \\
\hline Trimma unisquamis & 29 & $0.44-0.86$ & 1.4097 & 19.7362 & 0.84 \\
\hline \multicolumn{6}{|l|}{ Acanthuroidei } \\
\hline \multicolumn{6}{|l|}{ Acanthuridae } \\
\hline Acanthurinae & 43 & $1.43-5.21$ & -9.3721 & 29.2571 & 0.77 \\
\hline Acantburus $^{k}$ & 28 & $2.32-5.08$ & -23.0534 & 34.4433 & 0.61 \\
\hline nigrofuscus & 7 & $3.24-4.81$ & -13.3996 & 26.6374 & 0.91 \\
\hline triostegus & 12 & $2.66-3.88$ & -87.1491 & 60.8449 & 0.93 \\
\hline Ctenochaetus strigosus & 10 & $3.80-5.21$ & -43.8559 & 33.2044 & 0.81 \\
\hline Zebrasoma flavescens & 5 & $1.43-3.88$ & -30.9091 & 41.7762 & 0.97 \\
\hline \multicolumn{6}{|l|}{ Tetraodontiformes } \\
\hline \multicolumn{6}{|l|}{ Tetraodontoidei } \\
\hline \multicolumn{6}{|l|}{ Tetraodontidae } \\
\hline \multicolumn{6}{|l|}{ Canthigasterinae } \\
\hline Canthigaster (amboinensis, coronata) & 17 & $0.76-1.30$ & -6.7212 & 56.7368 & 0.67 \\
\hline jactator & 12 & $0.87-1.24$ & -7.1636 & 54.9559 & 0.75 \\
\hline
\end{tabular}

${ }^{a}$ G. eurostus, G. flavimarginatus, G. melatremus, G. meleagris, G. undulatus.

${ }^{b}$ S. dermatogenys, S. ulae, S. usitatus.

${ }^{c} S$. diadema, S. punctatissimum, S. spiniferum.

${ }^{d}$ Carangoides orthogrammus, Caranx ignobilis, C. melampygus, Decapterus macarellus, Scomberoides lysan, Seriola dumerili.

${ }^{e}$ Forcipiger flavissimus, F. longirostris, Hemitaurichthys polylepis, Heniochus diphreutes.

${ }^{f}$ C. fremblii, C. kleinii, C. lunula, C. lunulatus, C. miliaris, C. ornatissimus, C. quadrimaculatus, C. unimaculatus.

g Oxycheilinus bimaculatus, Pseudocheilinus evidanus.

${ }^{b}$ Gomphosus varius, Halichoeres ornatissimus, Macropharyngodon geoffroy, Pseudojuloides cerasinus, Stethojulis balteata.

${ }^{i}$ P. imparipennis, $P$. jobnstonianus.

${ }^{j}$ Enchelyurus brunneolus, Plagiotremus ewaensis, P. goslinei.

${ }^{k}$ A. leucopareius, A. nigroris, A. olivaceus, A. xanthopterus. 
Appendix 2

Power Functions for Otolith Length (OL) in millimeters versus Total Body Weight (wt) in grams $\left[w t=a(\mathrm{OL})^{b}\right]$

\begin{tabular}{|c|c|c|c|c|c|}
\hline Taxon & $n$ & Range (mm) & $a$ & $b$ & $r^{2}$ \\
\hline \multicolumn{6}{|l|}{ Anguilliformes } \\
\hline \multicolumn{6}{|l|}{ Muraenidae } \\
\hline Gymnothorax ${ }^{a}$ & 17 & $1.48-6.03$ & 14.4055 & 3.0224 & 0.69 \\
\hline \multicolumn{6}{|l|}{ Aulopiformes } \\
\hline \multicolumn{6}{|l|}{ Synodontidae } \\
\hline Synodus (dermatogenys, ulae, usitatus) & 4 & $0.62-1.09$ & 1.1265 & 4.7862 & 1.00 \\
\hline \multicolumn{6}{|l|}{ Beryciformes } \\
\hline Holocentridae (Myripristis berndti, M. kuntee) & 20 & $4.02-9.66$ & 2.1109 & 1.9932 & 0.26 \\
\hline Sargocentron (punctatissimum, spiniferum) & 10 & $4.02-8.85$ & 0.0024 & 5.6832 & 0.99 \\
\hline diadema & 6 & $5.32-6.24$ & 0.0446 & 3.9981 & 0.71 \\
\hline \multicolumn{6}{|l|}{ Gasterosteiformes } \\
\hline \multicolumn{6}{|l|}{ Aulostomidae } \\
\hline Aulostomus chinensis & 11 & $1.76-2.47$ & 0.8205 & 7.0540 & 0.69 \\
\hline \multicolumn{6}{|l|}{ Scorpaeniformes } \\
\hline \multicolumn{6}{|l|}{ Scorpaenidae } \\
\hline Scorpaeninae (Scorpaenopsis cacopsis) & 61 & $1.17-11.95$ & 1.7142 & 2.9535 & 0.97 \\
\hline Scorpaenodes (birsutus) $)^{b}$ & 33 & $1.17-3.00$ & & & 0.71 \\
\hline kelloggic ${ }^{c}$ & 27 & $1.17-2.47$ & & & 0.76 \\
\hline Sebastapistes (ballieui, fowleri) & 25 & $1.30-4.22$ & 0.0342 & 4.5055 & 0.99 \\
\hline coniorta & 9 & $2.62-4.22$ & 0.0371 & 4.4469 & 0.99 \\
\hline galactacma & 10 & $1.30-2.86$ & 0.0602 & 3.8583 & 0.97 \\
\hline \multicolumn{6}{|l|}{ Perciformes } \\
\hline \multicolumn{6}{|l|}{ Cirrhitoidei } \\
\hline Cirrhitidae (Cirrbitops fasciatus) & 65 & $1.70-7.17$ & 0.2186 & 3.9380 & 0.91 \\
\hline Amblycirrbitus bimacula & 18 & $1.70-2.78$ & 0.1509 & 3.8199 & 0.82 \\
\hline Cirrbitus pinnulatus & 17 & $4.01-7.17$ & 0.4187 & 3.5905 & 0.75 \\
\hline Paracirrbites & 27 & $1.74-5.32$ & 0.0026 & 6.7255 & 0.90 \\
\hline arcatus & 6 & $1.74-3.88$ & 0.2317 & 3.4181 & 0.97 \\
\hline forsteri & 21 & $2.41-5.32$ & 0.0024 & 6.7632 & 0.87 \\
\hline \multicolumn{6}{|l|}{ Percoidei } \\
\hline \multicolumn{6}{|l|}{ Apogonidae } \\
\hline Apogoninae (Pristiapogon kallopterus) & 8 & $1.34-3.78$ & 0.0603 & 2.7928 & 0.98 \\
\hline Apogon erythrinus & 7 & $1.34-3.56$ & 0.0511 & 2.9434 & 0.97 \\
\hline Serranidae & 73 & $0.88-10.34$ & 0.0216 & 4.9885 & 0.94 \\
\hline \multicolumn{6}{|l|}{ Anthiinae } \\
\hline Plectranthias nanus & 6 & $1.86-2.70$ & 0.0475 & 3.2849 & 0.76 \\
\hline Epinephelinae $^{d}$ & 67 & $0.88-10.34$ & 0.0216 & 4.9885 & 0.94 \\
\hline Cephalopholis argus & 57 & $6.02-10.34$ & 0.0219 & 4.9821 & 0.92 \\
\hline Carangidae $^{I}$ & 7 & $1.56-13.31$ & 14.5222 & 2.4626 & 0.88 \\
\hline \multicolumn{6}{|l|}{ Lutjanidae } \\
\hline Lutjanus kasmira & 15 & $7.58-9.97$ & 0.6549 & 2.5485 & 0.40 \\
\hline Mullidae & 63 & $2.27-5.14$ & 0.0584 & 5.9335 & 0.85 \\
\hline Mulloidichthys (flavolineatus, vanicolensis) & 4 & $3.12-4.74$ & 0.6340 & 3.9364 & 0.94 \\
\hline Parupeneus (insularis) & 59 & $2.27-5.14$ & 0.0603 & 5.9518 & 0.91 \\
\hline cyclostomus & 9 & $2.37-5.14$ & 0.0084 & 7.1835 & 0.97 \\
\hline multifasciatus & 26 & $2.48-4.13$ & 1.1399 & 3.7126 & 0.56 \\
\hline pleurostigma & 4 & $2.27-4.17$ & 0.2976 & 4.8780 & 0.99 \\
\hline porphyreus & 17 & $2.41-4.63$ & 0.9420 & 4.0462 & 0.81 \\
\hline Chaetodontidae $^{f}$ & 35 & $2.32-5.43$ & 0.1968 & 4.1501 & 0.91 \\
\hline Chaetodong & 27 & $3.29-5.43$ & 0.1907 & 4.1700 & 0.91 \\
\hline multicinctus & 8 & $3.33-4.01$ & 7.1037 & 1.3805 & 0.51 \\
\hline \multicolumn{6}{|l|}{ Labroidei } \\
\hline \multicolumn{6}{|l|}{ Labridae } \\
\hline Bodianus albotaeniatus & 19 & $4.36-8.19$ & 1.5694 & 3.1149 & 0.64 \\
\hline Cheilinina $^{b}$ & 4 & $0.83-2.89$ & 0.3264 & 3.5396 & 0.98 \\
\hline Corinae $^{i}$ & 54 & $0.46-5.06$ & 2.0786 & 3.5165 & 0.83 \\
\hline
\end{tabular}


Appendix 2 (continued)

\begin{tabular}{|c|c|c|c|c|c|}
\hline Taxon & $n$ & Range (mm) & $a$ & $b$ & $r^{2}$ \\
\hline Anampses cuvier & 5 & $2.57-3.84$ & 1.4503 & 4.2652 & 0.96 \\
\hline Coris ( flavovittata, gaimard) & 14 & $1.56-4.43$ & 5.9694 & 2.7620 & 0.81 \\
\hline venusta & 10 & $1.69-2.26$ & 0.5245 & 4.7119 & 0.81 \\
\hline Thalasomma & 30 & $0.46-5.06$ & 0.4435 & 4.5112 & 0.90 \\
\hline ballieui & 18 & $2.06-5.05$ & 0.6992 & 4.2145 & 0.82 \\
\hline duperrey & 11 & $0.46-3.44$ & 3.6409 & 2.2297 & 0.85 \\
\hline \multirow{2}{*}{\multicolumn{6}{|c|}{$\begin{array}{c}\text { Pomacentridae } \\
\text { Chrominae }\end{array}$}} \\
\hline & & & & & \\
\hline Chromis (hanui, ovalis) & 23 & $1.24-6.75$ & 0.9579 & 2.3661 & 0.78 \\
\hline vanderbilti & 18 & $1.24-2.47$ & 0.0843 & 4.1649 & 0.86 \\
\hline Dascyllus albisella & 7 & $3.57-3.95$ & 0.0651 & 4.8391 & 0.73 \\
\hline Pomacentrinae (Stegastes marginatus) & 14 & $1.76-5.64$ & 0.0984 & 4.3794 & 0.94 \\
\hline Abudefduf abdominalis & 8 & $4.66-5.64$ & 0.5028 & 3.4101 & 0.70 \\
\hline Plectroglyphidodon ${ }^{j}$ & 6 & $1.76-3.41$ & 0.2636 & 3.3531 & 0.97 \\
\hline Scaridae $\mathrm{e}^{\mathrm{OJ}}$ & 6 & $4.63-8.40$ & 6.5119 & 3.0414 & 0.85 \\
\hline \multirow{2}{*}{\multicolumn{6}{|c|}{$\begin{array}{l}\text { Trachinoidei } \\
\text { Pinguipedidae }\end{array}$}} \\
\hline & & & & & \\
\hline Parapercis schauinslandii & 7 & $2.79-3.68$ & 0.0991 & 3.7647 & 0.90 \\
\hline${\text { Blennioidei } i^{i}}^{2}$ & 115 & $0.23-2.09$ & 0.1232 & 6.2899 & 0.77 \\
\hline \multicolumn{6}{|l|}{ Blenniidae } \\
\hline Salariini (Exallias brevis, Cirripectes quagga) & 57 & $0.41-2.09$ & 0.2575 & 5.2060 & 0.76 \\
\hline Cirripectes vanderbilti & 22 & $0.79-2.09$ & 0.7362 & 3.1971 & 0.97 \\
\hline Entomacrodus strasburgi & 32 & $0.41-0.85$ & 0.7141 & 3.2908 & 0.87 \\
\hline \multicolumn{6}{|l|}{ Tripterygiidae } \\
\hline Enneapterygius atriceps & 57 & $0.30-1.45$ & 0.1281 & 2.4773 & 0.90 \\
\hline \multicolumn{6}{|l|}{ Gobioidei } \\
\hline Gobiidae & 128 & $0.26-2.55$ & 0.1330 & 2.3381 & 0.96 \\
\hline Gobiinae (Coryphopterus duospilus) & 127 & $0.26-2.55$ & 0.1333 & 2.3363 & 0.96 \\
\hline Eviota (susanae) & 84 & $0.26-0.62$ & 0.2772 & 2.9737 & 0.54 \\
\hline epiphanes & 73 & $0.26-0.62$ & 0.2655 & 2.7889 & 0.76 \\
\hline Priolepis (farcimen) & 11 & $0.45-0.97$ & 0.0997 & 3.2523 & 0.76 \\
\hline eugenius & 6 & $0.45-0.96$ & 0.1099 & 3.1213 & 0.88 \\
\hline Trimma unisquamis & 30 & $0.44-0.86$ & 0.1853 & 3.6008 & 0.27 \\
\hline \multicolumn{6}{|l|}{ Acanthuroidei } \\
\hline \multicolumn{6}{|l|}{ Acanthuridae } \\
\hline Acantburus $^{m}$ & 20 & $2.32-5.08$ & 3.2123 & 2.4737 & 0.32 \\
\hline nigrofuscus & 6 & $3.34-4.56$ & 0.3143 & 3.4034 & 0.74 \\
\hline triostegus & 7 & $2.66-3.88$ & 0.1381 & 5.1965 & 0.76 \\
\hline Ctenochaetus strigosus & 9 & $3.80-5.21$ & 0.4840 & 3.3061 & 0.71 \\
\hline \multicolumn{6}{|l|}{ Tetraodontiformes } \\
\hline \multirow{2}{*}{\multicolumn{6}{|c|}{$\begin{array}{l}\text { Tetraodontoidei } \\
\text { Tetraodontidae }\end{array}$}} \\
\hline & & & & & \\
\hline \multicolumn{6}{|l|}{ Canthigasterinae } \\
\hline Canthigaster (amboinensis, coronata) & 17 & $0.76-1.30$ & 8.2161 & 3.3290 & 0.56 \\
\hline jactator & 12 & $0.87-1.24$ & 7.0020 & 3.1480 & 0.58 \\
\hline
\end{tabular}

a G. eurostus, G. flavimarginatus, G. melatremus, G. meleagris, G. undulatus.

${ }^{b} \mathrm{Wt}=0.1150+0.0325(\mathrm{OL})^{3.6323}$.

${ }^{c} \mathrm{Wt}=-0.0469+0.0602(\mathrm{OL})^{3.2297}$.

${ }^{d}$ Liopropoma collettei, Pseudogramma polyacanthum.

e Carangoides orthogrammus, Caranx ignobilis, C. melampygus, Decapterus macarellus, Scomberoides lysan, Seriola dumerili.

${ }^{f}$ Forcipiger flavissimus, F. longirostris, Hemitaurichthys polylepis, Heniochus diphreutes.

${ }^{g}$ C. fremblii, C. kleinii, C. lunula, C. lunulatus, C. miliaris, C. ornatissimus, C. quadrimaculatus, C. unimaculatus.

${ }^{b}$ Oxycheilinus bimaculatus, Pseudocheilinus evidanus.

${ }^{i}$ Gomphosus varius, Halichoeres ornatissimus, Macropharyngodon geoffroy, Pseudojuloides cerasinus, Stethojulis balteata.

${ }^{j}$ P. imparipennis, P. jobnstonianus.

${ }^{k}$ Calotomus carolinus, Chlorurus perspicillatus, C. spilurus.

${ }^{\prime}$ Enchelyurus brunneolus, Plagiotremus ewaensis, P. goslinei

${ }^{m}$ A. leucoparieus, A. nigroris, A. olivaceus, A. xanthopterus. 
